higher in the AI group than the non-AI group $(50.5 \%$ vs 35.5\%, P-value 0.039*) (IDDF2021-ABS-0048 Figure 2. ADR between groups).

Conclusions AI CLN is useful to detect polyp and can be an extra-pair of eyes during procedures to improve the PDR.

\section{IDDF2021-ABS-0049 A STUDY OF NEUROLOGICAL OUTCOMES IN PREMATURE BABIES BORN AT 24-32 WEEK GESTATION DEPENDING ON WHETHER THEY DID OR DID NOT HAVE NECROTIZING ENTEROCOLITIS}

Sabrina Tee*. Barts and the London, UK

\subsection{6/gutjnl-2021-IDDF.119}

Background Necrotizing enterocolitis (NEC) is unfortunately one of the most common acquired gastrointestinal diseases mostly affecting preterm ( $<37$ weeks gestation) neonates, and recent research has linked this disease to adverse neurological outcomes up to several years later. Despite significant advances in neonatal care, NEC still carries a high morbidity and mortality risk. Well-documented complications of babies who survive NEC include intestinal failure associated with liver disease and short bowel syndrome. This paper explores the neurological impact of NEC on psychomotor and cognitive abilities, and the incidence of cerebral palsy.

Methods An analysis of peer-reviewed literature including PubMed, Cochrane database and Embase was performed. The search strategies included the following keywords: necrotizing enterocolitis, necrotising enterocolitis, neurological outcome, neurodevelopment and neurol*. Citation searches were performed on Mendeley. Inclusion criteria were as follows: any study type, gestational age 24-32 weeks, studies published in the last 20 years (2000-2020), any birth weight, and followup after at least 12 months.

Results Most studies carried out neurological assessments at 18-22 months found an increased incidence of psychomotor impairment in the NEC babies, but there were varying results in terms of the degree of impairment that NEC put them at risk of. Similarly, the studies found that NEC, as opposed to noNEC was more likely to be associated with cognitive impairment. While smaller studies noted no increased risk of cerebral palsy in very low birth weight NEC patients, other larger systematic reviews revealed an increased risk of the permanent neurological condition.

Conclusions The study of various aspects of neurodevelopment in premature infants affected by NEC yields contradictory results. Most studies support the link between NEC and psychomotor impairment; however, the results differ when it comes to cognitive development and incidence of cerebral palsy. Even with the hazy link between NEC and psychomotor impairment, the extent or severity of retardation is still unclear. Prematurity is on its own associated with neurological impairment, and the degree to which NEC adds to this risk is not well defined. However, most studies agree that the presence of NEC is to some extent associated with adverse neurodevelopmental outcomes.

\section{IDDF2021-ABS-0052 CHINESE HERBAL MEDICINE FOR FUNCTIONAL DYSPEPSIA: NETWORK META-ANALYSIS OF PLACEBO- CONTROLLED RANDOMISED TRIALS}

${ }^{1}$ Leonard Ho* ${ }^{2}$ Claire Chenwen Zhong, ${ }^{2}$ Charlene Hoi Lam Wong, ${ }^{3}$ Justin Che Yuen Wu, ${ }^{4}$ Karina Kar-Han Chan, ${ }^{5}$ Irene Xinyin Wu, ${ }^{1}$ Ting Hung Leung, ${ }^{6}$ Vincent Chi Ho Chung. ${ }^{1}$ School of Chinese Medicine, Faculty of Medicine, The Chinese University of Hong Kong, Hong Kong; ${ }^{2}$ The Jockey Club School of Public Health and Primary Care, Faculty of Medicine, The Chinese University of Hong Kong, Hong Kong; ${ }^{3}$ Department of Medicine and Therapeutics, Faculty of Medicine, The Chinese University of Hong Kong, Hong Kong; ${ }^{4}$ United Christian Nethersole Community Health Service - The Chinese University of Hong Kong Chinese Medicine Clinic cum Training and Research Centre (Tai Po District), Hong Kong; ${ }^{5}$ Xiangya School of Public Health, Central South University, Hunan, China; ${ }^{6}$ School of Chinese Medicine/The Jockey Club School of Public Health and Primary Care, Faculty of Medicine, The Chinese University of Hong Kong, Hong Kong

\subsection{6/gutjpl-2021-IDDF.120}

Background Functional dyspepsia (FD) is a common functional gastrointestinal disorder, having a prevalence of $10-40 \%$ in Western countries and $5-30 \%$ in Asia. Conventional treatments for FD are limited and associated with bothersome adverse events. Chinese herbal medicine (CHM) use is prevalent despite unclear comparative effectiveness among different formulae. This network meta-analysis (NMA) aimed to evaluate the comparative effectiveness of CHM formulae for FD against placebo.

Methods Three international (MEDLINE, EMBASE, and Cochrane Central Register of Controlled Trials) and four Chinese (Wanfang Data, China National Knowledge Infrastructure, SinoMed, and Index to Taiwan Periodical Literature System) databases were searched for randomised controlled trials (RCTs) on CHM versus placebo. The risk of bias among RCTs was assessed using Cochrane Risk-of-Bias Tool 2. Data from RCTs were extracted for random-effect pairwise metaanalyses. Random-effect NMAs were performed to evaluate the comparative effectiveness of CHM formulae. GRADE partially contextualised framework was adopted to facilitate NMA result interpretation.

Results Twelve different CHM formulae were identified from fifteen RCTs of mediocre quality. At 8-week follow-up, pairwise meta-analyses indicated that $\mathrm{CHM}$ was superior to placebo in alleviating global symptoms (pooled risk difference (RD): $0.20 ; 95 \%$ confidence interval (CI): 0.11-0.29), with effect size larger than the minimally clinically important difference of $0.20 \mathrm{RD}$. Sensitivity analysis showed no significant impact on results attributable to the risk of bias. NMAs demonstrated that Xiao Yao Pill and Modified Ban Xia Xie Xin Decoction probably have a large beneficial effect on alleviating global symptoms (RD: 0.37 ; 95\% CI: 0.03-0.99) and postprandial fullness (standardised mean difference: -0.93; 95\% credible interval: -1.61- -0.06), respectively. No serious adverse events were reported.

Conclusions Xiao Yao Pill and Modified Ban Xia Xie Xin Decoction may be considered as an alternative among patients unresponsive to conventional treatments. The comparative effectiveness of these two formulae should be evaluated in future head-to-head confirmatory trials. 